\title{
Improving Robustness and Precision in Mobile Robot Localization by Using Laser Range Finding and Monocular Vision
}

\author{
Kai O. Arras, Nicola Tomatis \\ Autonomous System Lab \\ Swiss Federal Institute of Technol ogy Lausanne (EPFL) \\ $\mathrm{CH}-1015$ Lausanne, Switzerland \\ kai-oliver.arras@epfl.ch, nicola.tomatis@epfl.ch
}

\begin{abstract}
This paper discusses mobile robot localization by means of geometric features from a laser range finder and a CCD camera. The features are line segments from the laser scanner and vertical edges from the camera. Emphasis is put on sensor models with a strong physical basis. For both sensors, uncertainties in the calibration and measurement process are adequately modeled and propagated through the feature extractors. This yields observations with their first order covariance estimates which are passed to an extended Kalman filter for fusion and position estimation.

Experiments on a real platform show that opposed to the use of thelaser rangefinder only, the multisensor setup allows the uncertainty to stay bounded in difficult localization situations like long corridors and contributes to an important reduction of uncertainty, particularly in the orientation. The experiments further demonstrate the applicability of such a multisensor localization system in real-time on a fully autonomous robot.
\end{abstract}

\section{Introduction}

Localization in a known, unmodified environment belongs to the basic skills of a mobile robot. In many potential service applications of mobile systems, the vehicle is operating in a structured or semi structured surrounding. This property can be exploited by using these structures as frequently and reliably recognizable landmarks for navigation. Topological, metric or hybrid navigation schemes make use of different types of environment features on different levels of perceptual abstraction.

Raw data have the advantage of being as general as possible. But, with most sensors, they are credible only by processing great amounts and are of low informative value when looking for concise scene descriptions. Navigation based on geometric features allow for compact and precise environment models. Maps of this type are furthermore directly extensible with feature information from different sensors and thus a good choice for multisensor navigation. This approach relies however on the existence of features which represents a limitation of environment types.

This is viewed as a loss of robustness which can be diminished by simultaneously employing geometric features from different sensors with complementary properties. In this work we consider navigation by means of line segments extracted from 1D range data of a $360^{\circ}$ laser scanner and vertical edges extracted from images of an embarked CCD camera.

Precise localization is important in service tasks where load stations might demand accurate docking maneuvers. Mail delivery is such an example [2]. When the task includes operation in crowded environments where a moving vehicle is supposed to suggest reliability and predictability, precise and thus repeatable navigation helps evoking this subjective impression.

The use of the Kalman filter for localization by means of line segments from range data is not new [9][11][2][7]. Vertical edges have been equally employed [8], and propositions for specific matching strategies are available in this context [12]. In [10], the same features were applied for approaching the relocation problem. The multisensor setup was used to validate observations of both sensors before accepting them for localization. In [13], a similar setup was used with a 3D laser sensor simultaneously delivering range and intensity images of the scene in front of the robot. Line segments and vertical edges were also employed in a recent work [14], where the localization precision of laser, monocular and trinocular vision has been separately examined and compared to ground truth measurements. 


\section{Sensor Modeling}

It is attempted to derive uncertainty models of the sensors employed with a strong physical basis. Strictly speaking, it is necessary to trace each source of uncertainty in the measurement process and, with knowledge of the exact measurement principle, propagate it through the sensor electronics up to the raw measurement the operator will see. This allows for a consequent statistical treatment with noise models of high fidelity which is of great importance for all subsequent stages like feature extraction and matching.

\subsection{Laser Range Finder}

In all our experiments we used the commercially available Acuity AccuRange4000LR. The Acuity sensor is a compromise between building a laser range finder by one's own and devices like the scanners of SICK (e.g. PLS100, LMS200). The latter two deliver both range and angle information and come with standard interfaces. Besides the protocol driver which is to be written, they can be used practically plug-and-play. The disadvantage is that this black-box character inhibits the abovementioned analysis of noise sources. The AccuRange 4000 provides range, amplitude and sensor temperature information, where amplitude is the signal strength of the reflected laser beam. They are available as anal ogue signals.

Relationships for range and angle variance are sought. The accuracy limit of encoders are usually low with respect to the beam spot size. Angular variability is therefore neglected. For range accuracy there are several factors which influence the extent of noise:

- The amplitude of the returned signal which is available as measurement.

- Drift and fluctuations in sensor circuitry. At the configured sampling frequency $(1 \mathrm{kHz})$ this is predominant over thermal noise of the detection photodiode and resolution artifacts of the internal timers.

- Noise injected by the AD conversion electronics.

In [1] a phase shift measurement principle has been examined yiel ding a range variance to amplitude relationship of the form $\sigma_{\rho}^{2}=a / V_{r}+b$, where $\sigma_{\rho}^{2}$ is range variance and $V_{r}$ the measured amplitude. After identification, an inverse, slightly nonlinear function was found. For identification in our case, an experiment was performed with a stationary target at about 1 meter distance. The returned signal strength was varied systematically with a
Kodak gray scale control patch where $10^{\prime} 000$ readings were taken at each of the twenty gray levels.

Opposed to the model in [1], we can observe an abrupt rise of noise below a certain amplitude (fig. 1). This reduces our model for range variance to a constant value, independent on target distance and amplitude.

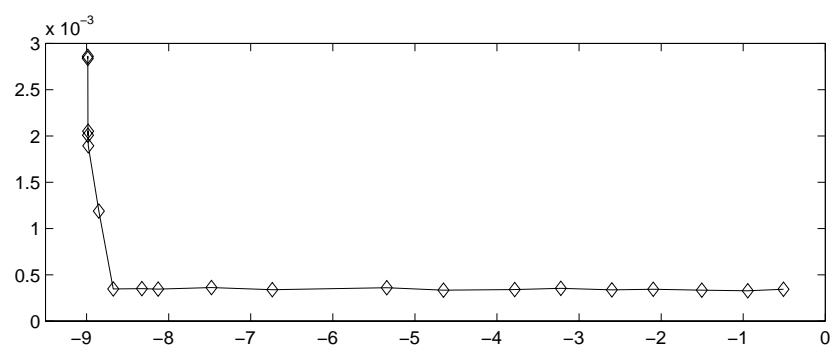

Figure 1: Range standard deviation (y-axis, in meters) against measured amplitude (x-axis, in Volts). 10 '000 readings, measurements were done with a Kodak gray scale control patch.

Although this analysis lead to such a simple result it permits rejection of false or very uncertain readings by means of the amplitude measurements. This is very important since in many practical cases the sensor exhibits strong dependency upon the surface properties like color and roughness. Moreover, the Acuity sensor is often and reproducibly subject to outliers. When the laser beam hits no target at all, and at normally occurring range discontinuities it returns an arbitrary range value, typically accompanied by a low signal strength.

\subsection{CCD Camera}

The vision system consists in a Pulnix TM-9701 full frame, gray-scale, EIA $(640 \times 480)$ camera with an effective opening angle of $54^{\circ}$ which sends a standard RS-170 signal to a Bt848 based frame grabber. No dedicated DSPs are used in our setup, all image processing is done directly by the CPU of the VME card.

A vision task which is intended to extract accurate geometric information from a scene requires a calibrated vision system. For this a variety of camera parameters including its position and orientation (extrinsic parameters), image center, scale factor, lens focal length (intrinsic parameters) and distortion parameters are to be determined. To calculate a coherent set of intrinsic, extrinsic and distortion parameters the calibration technique [15] has been combined with a priori knowledge of features from a test field. The procedure is to extract 


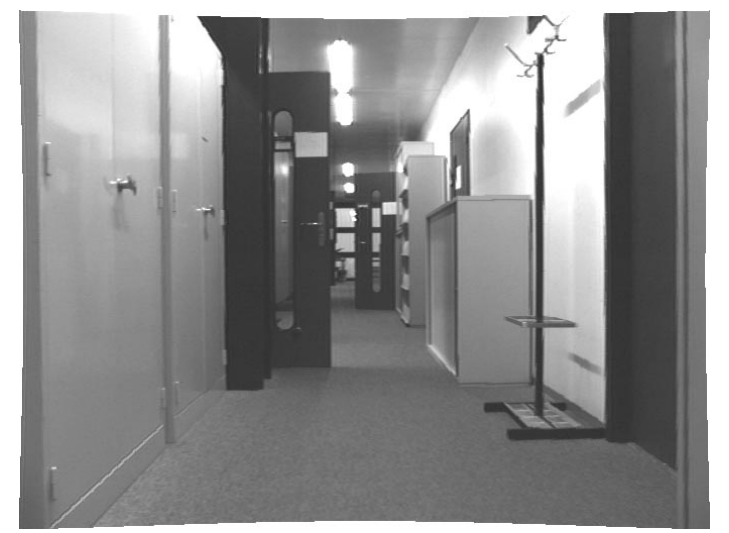

Figure 2: CCD image of the corridor where the experiments havebeen carried out (step 5 of thetrajectory). The image is compensated for radial distortion.

and fit vertical and horizontal lines from the test field and determine the distortion in the $x$ - and $y$ direction. By knowing the 3D position of these lines in space, the intrinsic, extrinsic and distortion parameters can be determined simultaneously.

Due to the particularity of our application, some simplifications for the final calibration model can be done. The camera is mounted horizontally on the robot and the center of projection of the imaging device is at $(0,0)$ in robot coordinates with orientation $0^{\circ}$. Since only vertical edges are extracted, calibration is needed only in the horizontal direction (x-axis). With that, only few parameters remain to be modeled: the focal length $\mathrm{C}$, the image center $\left(c_{x}, c_{y}\right)$, and the distortion parameters $k_{1}, k_{2}$. This yields equation (1) for parameter definition

$$
C \cdot \frac{x_{w}}{z_{w}}=x^{\prime}+x_{c}\left(k_{1} r^{2}+k_{2} r^{4}\right),
$$

where $x^{\prime}$ refers to the distorted location, $x_{c}$ $=x^{\prime}-c_{x}, y_{c}=y^{\prime}-c_{y}, r^{2}=x_{c}^{2}+y_{c}^{2}$ and $x_{w}, z_{w}$ are measures of the test field in camera coordinates. The angle $\varphi$ of a feature relative to the robot is finally calculated using equations (2) and (3).

$$
\begin{gathered}
\bar{x}=x^{\prime}+x_{c}\left(k_{1} r^{2}+k_{2} r^{4}\right) \\
\varphi=\operatorname{atan}(\bar{x} / C)
\end{gathered}
$$

The uncertainties from the test field geometry and those caused by noise in the camera electronics and frame grabber AD conversion are propagated through the camera calibration procedure onto the level of camera parameters yielding a $4 \times 4$ parameter covariance matrix.

\section{Feature Extraction}

Geometric environment features can describe structured environments at least partially in a compact and exact way. Horizontal or vertical line segments are of high interest due to the frequent occurrence of line-like structures in man-made environments and the simplicity of their extraction. More specifically, the problem of fitting a model to raw data in the least squares sense has closed form solutions if the model is a line. This is even the case when geometrically meaningful errors are minimized, e.g. the perpendicular distances from the points to the line. Already slightly more complex models like circles do not have this property anymore.

\subsection{Laser Range Finder}

The extraction method for line segments from 1D range data has been described in [3]. A short outline of the algorithm shall be given.

The method delivers lines and segments with their first order covariance estimate using polar coordinates. The line model is

$$
\rho \cos (\varphi-\alpha)-r=0
$$

where $(\rho, \varphi)$ is the raw measurement and $(\alpha, r)$ the model parameters.

Segmentation is done by a relative model fidelity measure of adjacent groups of points. If these groups consist of model inliers they constitute a compact cluster in model space since their parame ter vectors of the previously fitted model are similar. Segments are found by comparing this criterion against a threshold. A hierarchical agglomerative clustering algorithm with a Mahalanobis distance matrix is then applied. It merges adjacent segments until their distance in model space is greater than a threshold.

The constraint of neighborhood is removed as soon as the threshold has been exceeded so as to fuse non-adjacent segments as well. The clustering terminates if the same threshold was reached again. This allows for particular precise reestimations of spatially extended structures like for example a long wall where objects, doors or other discontinuities lead to multiple small segments belonging to this wall.

For the line fitting, the nonlinear regression equations have been explicitly derived for polar coordinates minimizing the weighted perpendicular error from the points to the line. Opposed to a Kalman filter, more general error scenarios of pos- 
sibly correlated measurements can be taken into account.

An advantage of this method is its generality. This follows from the fact that all decisions on segmentation and merging are taken in the model space, independent on the spatial appearance of the model. Only the fitting step is inherently model specific and has to provide the parameter's first and second moments.

In [7] the segmentation method first detects homogeneous regions which are basically marked by unexpected range discontinuities. Identical to the detection of edges in [1], this is done with a Kalman filter. Afterwards segments are found by a widely used recursive algorithm which applies a distance criterion from the points to their current homogeneous region. If the maximal distance is above a threshold, the segment is splitted into two separate regions restarting the process for them. The line parameter estimate is finally done with an information filter. Alike [3] consecutive segments are merged and reestimated if their moments exhibit sufficient similarity. In [16] range discontinuities are equally detected with a Kalman filter. They serve directly as segment end points.

\subsection{CCD Camera}

If the camera has a horizontal position on the vehicle, vertical structures have the advantage of being view invariant, opposed to horizontal ones. Furthermore, they require simple image processing which is important for a real-time implementation under conditions of moderate computing power.

For several reasons, only the lower half of the image is taken: better illumination conditions (less direct light from windows and lamps), the availability of corresponding feature information to the one from the laser range finder and more frequent occurrence of reliable vertical edges.

The extraction steps can be summarized as follows:

- Vertical edge enhancement: Edge extraction using a specialized Sobel filter which approximates the image gradient in the $x$-direction.

- Non-maxima suppression with dynamic thresholding: The most relevant edge pixels (maximal gradient) are extracted and thinned by using a standard method.

- Edge image calibration: The camera model is applied to the edge image using the previously determined parameters. The image is calibrated only horizontally.
- Line fitting: Columns with a predefined number of edge pixel are labelled as vertical edges. Line fitting reduces to a 1D problem. Sub-pixel precision is achieved by calculating the weighted mean of the non-discrete edge pixel position after image calibration.
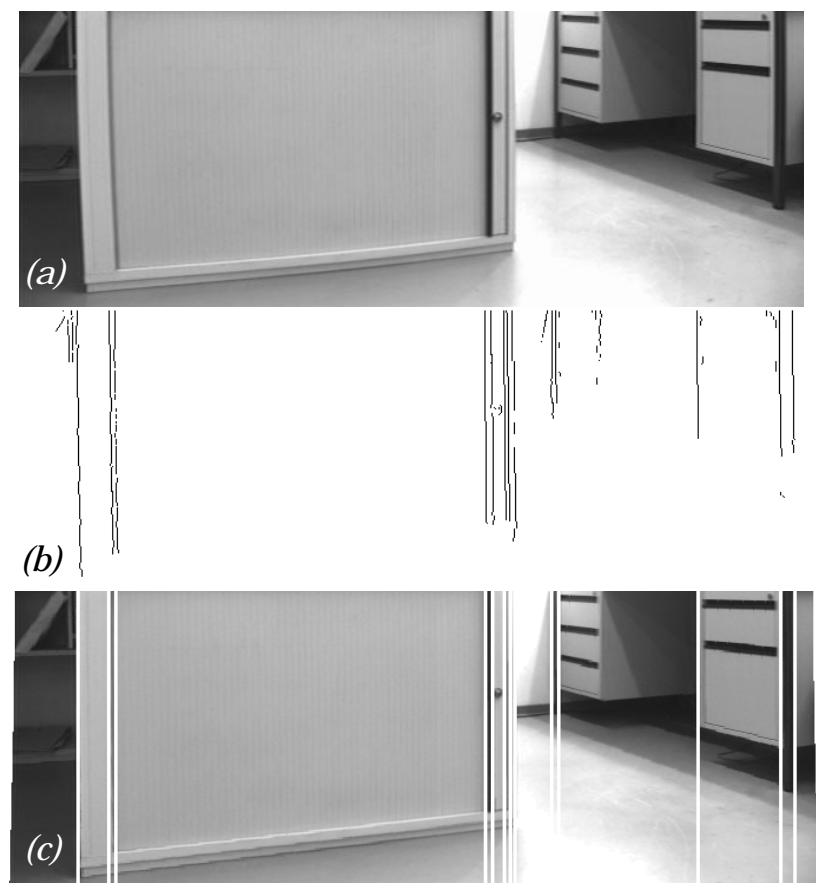

Figure 3: Extraction of vertical edges. (a): uncalibrated half image, (b): edge pixels after edge enhancement, non-maxima suppression and calibration, (c): resulting vertical edges.

This line extraction method is essentially a special case of the Hough transformation, where the model is one-dimensional since the direction is kept constant.

In each abovementioned step, the uncertainty of the calibration parameters and a constant uncertainty in the x-position of the input image are propagated. The result is a set of vertical edges described by their first two moments $\left(\varphi, \sigma_{\varphi}^{2}\right)$.

\section{EKF Localization}

Under the assumption of independent errors, the estimation framework of a Kalman filter can be extended with information from additional sensors in a straight-forward way. Since this paper does not depart from the usual use of extended Kalman filtering and error propagation based on first-order Taylor series expansion, most mathematical 
details are omitted. Only aspects which are particular are presented. Please refer to [2][3][5] or [11] for more profound treatments.

The prediction step uses an a priori map of the environment which has been measured by hand. Each feature $p_{i}$ in the map (i.e. lines and vertical edges) received constant values for their positional uncertainty, held by the covariance matrix ${ }^{W} C_{p}^{[i]}$, where superscript $\mathrm{W}$ denotes the world frame. Together with the predicted robot pose uncertainty $P(k+1 \mid k)$, it is propagated into the according sensor frame for prediction. Additionally, the covariance matrix ${ }^{R} C_{S}$ accounts for uncertain robot-tosensor frame transformations. The innovation covariance matrix of prediction $\hat{z}_{i}$ and observation $z_{j}$ at time index $k+1$ is then

$$
\begin{aligned}
S_{i j}(k+1)= & \nabla h_{x}^{[i]} \cdot P(k+1 \mid k) \cdot \nabla h_{x}^{[i]^{T}}+\nabla h_{p}^{[i]} \cdot{ }^{W} C_{p}^{[i]} \cdot \nabla h_{p}^{[i]^{T}} \\
& +\nabla h_{s}^{[i]} \cdot{ }^{R} C_{S} \cdot \nabla h_{s}^{[i]}+R_{j}(k+1),
\end{aligned}
$$

where $R_{j}(k+1)$ is the first order covariance estimate of observation $z_{j}, \nabla h_{x, p, s}^{[i]}$ the measurement J acobian with respect to the uncertain vectors $x, p, s$ of the nonlinear measurement model $h($.) relating the system state $x$ to the measurements for prediction $\hat{z}_{i}$.

For matching, an iterative strategy has been implemented where each step includes (i) matching of the current best pairing, (ii) estimation and (iii) re-prediction of features not associated so far. As in [13], line segments are integrated first since they are mutually more distinct, leading less often to ambiguous matching situations.

Always the current best match is searched among pairs of predictions $\hat{z}_{i}$ and observations $z_{j}$ which satisfy the validation test

$$
\left(z_{j}-\hat{z}_{i}\right) S_{i j}^{-1}\left(z_{j}-\hat{z}_{i}\right)^{T} \leq \chi_{\alpha, n}^{2}
$$

where $\chi_{\alpha, n}^{2}$ is a threshold reflecting a probability level $\alpha$ chosen from a chi-square distribution with $n$ degrees of freedom. Matching observed segments to predicted lines from the map is done in the $(\alpha, r)$-model space, therefore $n=2$. With vertical edges $n=1$ since the $\varphi$-model space is onedimensional.

The criterion of pairing quality is different for segments and vertical edges. 'Best' for line segments means smallest observational uncertainty among the candidates satisfying (6) - not smallest Mahalanobis distance. This renders the matching more robust against small spurious line segments which have been extracted in groups of outliers occasionally occurring with our sensor (chapter 2).
For several reasons vertical edges are much more difficult to associate [13][14]. For example, multiple, closely extracted vertical edges (see fig. 3) are often confronted with large validation regions around the predictions. Accordingly, 'best' for vertical edges means unique match candidate with smallest Mahalanobis distance. When there is no unique pairing anymore, candidates with multiple observations in the validation region are accepted and chosen according to the smallest Mahalanobis distance of the their closest observation.

\section{Implementation and Experiments}

\subsection{The Robot Pygmalion}

Our experimental platform is the robot Pygmalion which has been recently built in our lab (fig. 4). Its design principles are oriented towards an application as service or personal robot. Long-term autonomy, safety, extensibility, and friendly appearance were the main objectives for design. With its dimensions of about $45 \times 45 \times 70 \mathrm{~cm}$ and its weight of $55 \mathrm{~kg}$ it is of moderate size and danger opposed to many robots in its performance class. The system runs the deadline-driven, hard real-time operating system XOberon [6].

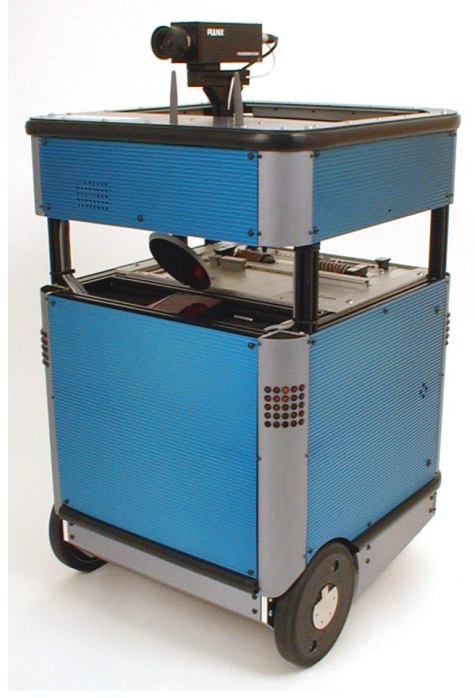

Figure 4: Pygmalion, the robot which was used in the experiments. It is a VME based system with a six axis robot controller. The processor board carries currently a PowerPC at $100 \mathrm{MHz}$. Besides wheel encoders and bumpers, the sensory system includes the laser range finder and the CCD camera discussed in the second chapter.

\subsection{Experimental Results}

Long corridors notoriously recur in mobile robotics. They are, however, an excellent benchmark for the localization system of a service robot, since they occur often in realistic application scenarios and contain the difficulty to stay localized in the 


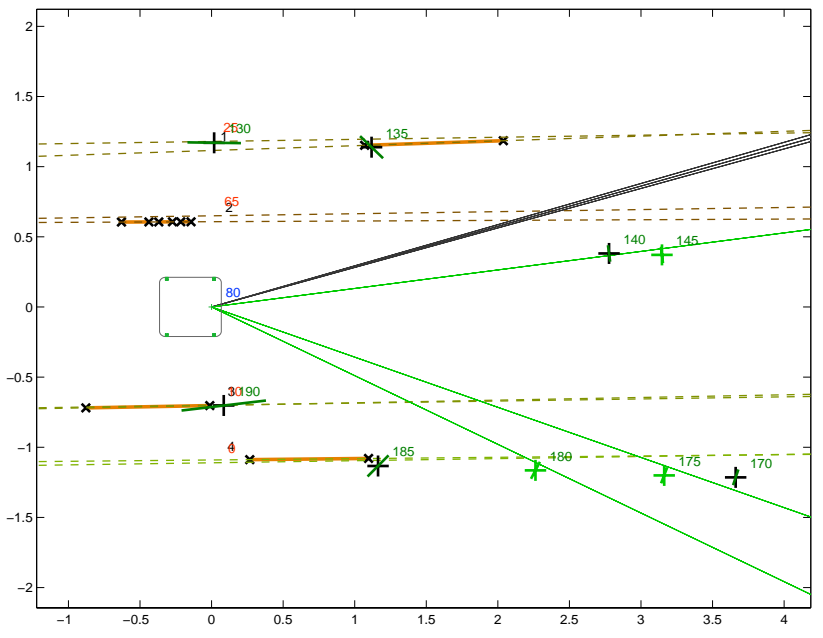

Figure 5: Predictions and observations in the robot frame beforeestimation at step 14 of thetest trajectory. All line segments and three vertical edges have been matched (to predictions 145, 175, 180).

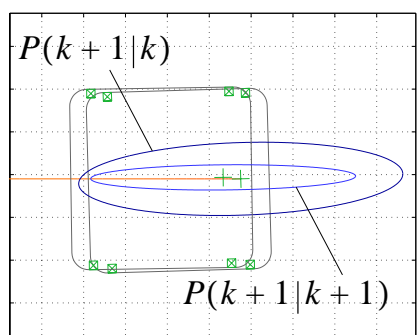

Figure 6: U pdateresulting from thematches in fig. 5. The Kalman filter corrects the robot about $5 \mathrm{~cm}$ backwards, establishing good correspondence of observation and prediction of thethrematched verti-

cal edges in fig. 5. Thus they allow for an update also in corridor direction; the state covariance matrix $P(k+1 \mid k+1)$ stays bounded.

direction of travel. In order to demonstrate the performance of our multisensor localization system, we chose the corridor environment shown in fig. 2, 7 and 8 as benchmark. Two experiments have been conducted where the robot drove an overall distance of more than $1 \mathrm{~km}$. In both experiments the vehicle followed the trajectory depicted in fig. 7 and 8 with a length of about $78 \mathrm{~m}$.

The robot moved autonomously in a stop-and-go mode, driven by a position controller for non-holonomic vehicles [4]. A graph with nodes at characteristic locations was built which allows for global planning in the map. No local navigation strategy accounting for unmodeled objects was active. Since the angle of the laser range finder has to be calibrated at each system startup by measuring the four vertical profiles (see fig. 4), there is a angular uncertainty of the robot-to-sensor frame transformation. This is modeled by setting ${ }^{R} C_{S_{l}}=\sigma_{\theta_{s}}^{2}$ with $\sigma_{\theta_{s}}$ reflecting $1.5^{\circ}$ in equation (5). For the vision system ${ }^{R} C_{S}$ is set to 0 , since this uncertain transformation is al ready captured by the camera calibration and its uncertain parameters (chapter 2.2).

From the figures it can be seen that the corridor is actually well-conditioned in the sense that many structures exist which can be used for updates in the direction of travel. In the first experiment all these structures have been removed from the map. Only in the two labs and at the upper corridor end the map was left unchanged. In fig. 7 and 8 modeled structures are in black, unmodeled ones in gray. It is attempted to demonstrate the need of additional environment information, if, due to a difficult working area, information of one type is not sufficient for accomplishing the navigation task.

Figure 7 shows one of three runs which were performed with the laser range finder only. As to be expected during navigation in the corridor, uncertainty growed boundless in the direction of travel. Inaccurate and uncertain position estimates lead regularly to false matches and incorrect pose updates in front of the lower lab.

In the second run of the first experiment (fig. 8, one of three runs shown), the vision system was able to ameliorate precision and reduce uncertainty to an uncritical extent. This is illustrated in fig. 5 and fig. 6, where for one of the corridor situations (step 14), predictions and extraction results are displayed in the robot frame before estimation. The three matched vertical edges allow for a full update. The task could always be finished. The precision at the endpoint was typically in a subcentimeter range, showing a slight temperature dependency of the Acuity sensor.

In the second experiment, the contribution of monocular vision to the reduction of estimation uncertainty has been examined. The same test path was travelled five times with the laser range finder only and five times with both sensors. The map was complete, i.e. no structures had been removed. The resulting averaged error bounds are shown in fig. 9. In fig. 10 the mean numbers of predictions, observations and matchings at the 47 steps of the test path are presented, and table 1 briefly quantifies the results.

The resulting error bounds in figure 9 and their overall means in table 1 show that, compared to the laser-only case, the multisensor system particularly contributes to the reduction of uncertainty in the vehicle orientation. This although the number of matched vertical edges is relatively low due to their infrequent occurrence in our map. 


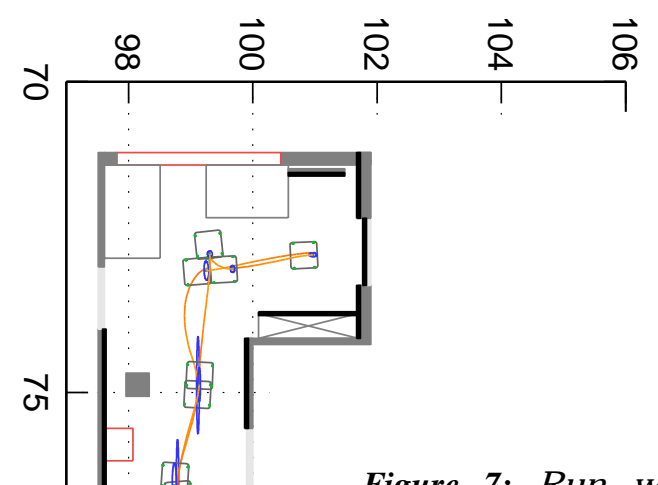

Figure 7: Run with laser range finder only. All lines which would allow for an update in thecorridor directions have been removed from the map. Modeled environment features are in black.

At each of the 47 trajectory steps, the robot is depicted with an ellipsereflecting the $99.9 \%$ probability region of the posterior state covariance matrix $P(k+1 \mid k+1)$ for $x$ and $y$. The axis dimension is meter.

The result is typical. False matches due to great position errors and extensive uncertainty at the lower corridor end yield incorrect estimates. The robot is lost.

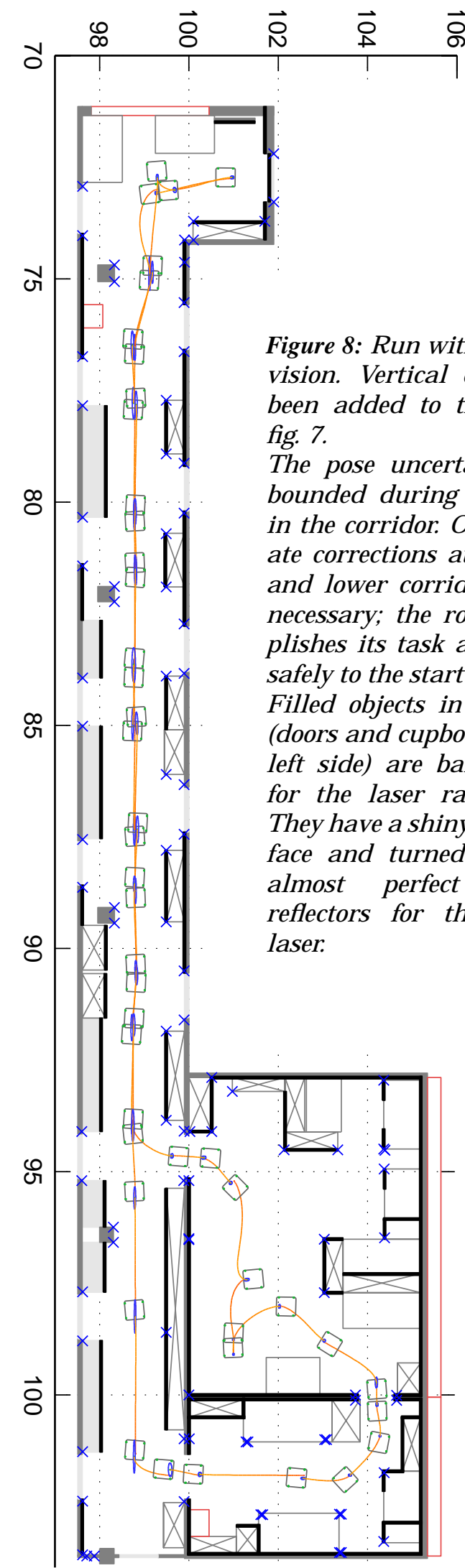

Proceedings of the Third European Workshop on Advanced Mobile Robots (EUROBOT ‘99), Zurich, Switzerland, Sept.6-8, 1999. 

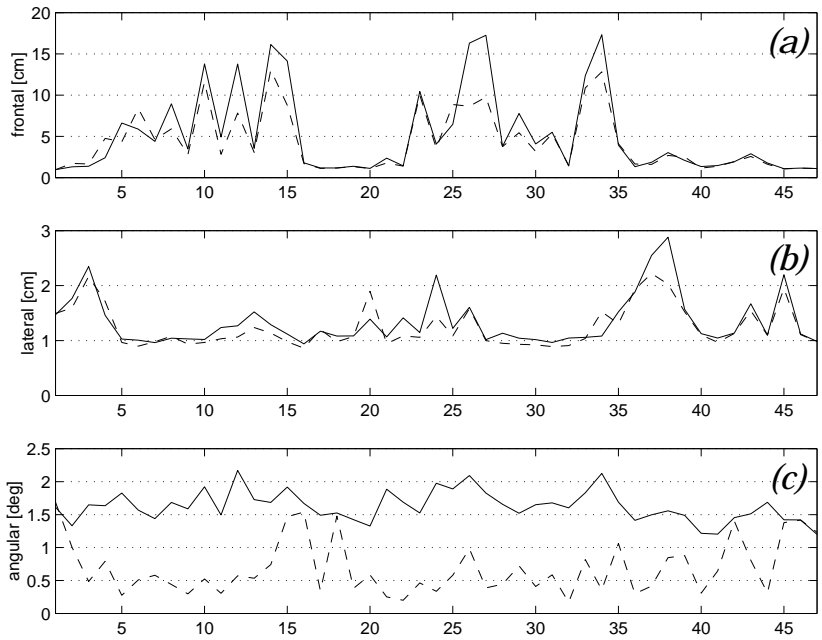

Figure 9: Averaged $2 \sigma$-error bounds of frontal (a), lateral (b) and angular (c) a posterior uncertainty during the 47 steps of the test trajectory. Five runs have been made for each mode Solid lines: laser range finder only, dashed lines: laser and vision.
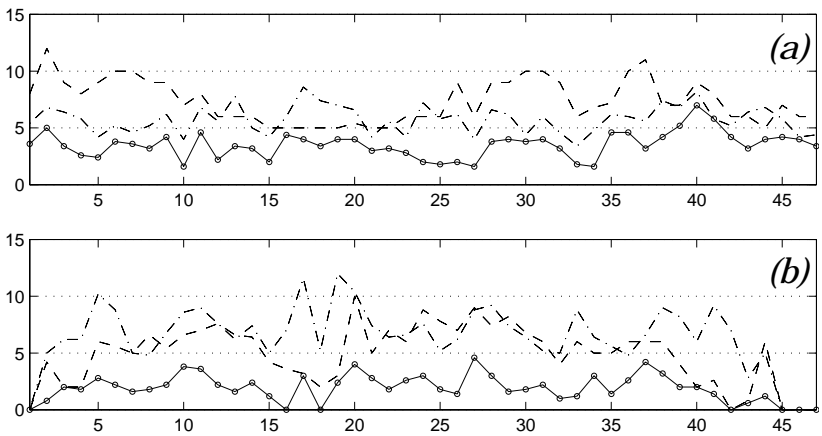

Figure 10: Averaged number of predictions (--), observations $(--)$ and matches $(-)$ for line segments (a) and vertical edges (b). Seealso table 1.

The average execution times express overall durations and reflect the full CPU load including sensor acquisition, low-level controllers and communication. No special code optimization has been done.

\section{Discussion}

Experience with earlier implementations [2] showed that on-the-fly localization during motion is not merely an option for good looking demonstrations. It augments the robustness against many factors because it brings the update rate towards a better ratio to the dynamics of the Kalman filter variables. Stop-and-go navigation with

\begin{tabular}{c|c|c}
\hline & laser & Iaser and vision \\
\hline \hline $2 \bar{\sigma}_{\text {frontal }}$ & $5.191 \mathrm{~cm}$ & $4.258 \mathrm{~cm}$ \\
\hline $2 \bar{\sigma}_{\text {lateral }}$ & $1.341 \mathrm{~cm}$ & $1.245 \mathrm{~cm}$ \\
\hline $2 \bar{\sigma}_{\text {angular }}$ & $1.625^{\circ}$ & $0.687^{\circ}$ \\
\hline $\bar{n}_{l} / \bar{n}_{v}$ & $3.53 /-$ & $3.51 / 1.91$ \\
\hline $\bar{t}_{\text {exe }}$ & $377 \mathrm{~ms}$ & $1596 \mathrm{~ms}$
\end{tabular}

Table 1: Overall mean values of the error bounds, the number of matched line segments $n_{l}$ and matched vertical edges $n_{v}$, and the average localization cycle time $\overline{t_{\text {exe }}}$.

long steps (like at the lower corridor end in fig. 7) causes very uncertain predictions, making the matching problem difficult for any feature. On the other hand, on-the-fly localization with more updates per distance travelled produces smaller validation regions and thus, diminishes the effect of a poorly calibrated odometry and an inadequate model for nonsystematic odometry errors.

Matching vertical edges is especially error-prone due to their frequent appearance in groups (fig. 3). With large validation regions caused by very uncertain pose predictions, false matches have been occasionally produced. But the effect of these incorrect assignments remain weak since these groups are typically very compact. A possible remedy is to exploit the prior knowledge from the map and to try to 'correlate' patterns of edges to the observation. Instead of independently matching single observations to single predictions, the observation pattern will be forced to match a similar constellation of predicted edges [10][12].

Furthermore, for the vision system, the large validation regions are contrasted by observations whose uncertainties are very small in comparison. From the experiments we conclude that it would be more relevant to model an uncertain robot-tosensor frame transformation to account for vibrations and uneven floors, i.e. alike ${ }^{R} C_{S_{l}}$ setting ${ }^{R} C_{S_{v}} \neq 0$ in equation (5).

\section{Conclusions and Outlook}

It could be demonstrated that the presented multisensor setup allows for more robust navigation in critical localization situations like long corridors. The permanent occurrence of features of a specific type becomes less important. The experiments showed furthermore that, compared to the laseronly case, already a moderate number of matched 
vertical edges contribute to an important reduction of estimation uncertainty, especially in the vehicle orientation.

By reducing the problem of camera calibration and edge extraction to an appropriate degree of specialization, the computational complexity could be kept low so as to have an applicable implementation on a fully autonomous system.

Further work will focus on more efficient implementations for on-the-fly localization during motion. Although ambiguous matching situation will be expected to appear less often, more sophisticated strategies could be envisaged [5][12]. Finally, more complex fusion scenarios of range and vision features could be addressed as well. Detection of high-level features for concise scene descriptions [3] can be a starting point for topology-based navigation schemes.

\section{References}

[1] Adams M.D., Optical Range Analysis of StableTarget Pursuit in Mobile Robotics. PhD Thesis, University of Oxford, UK, Dec. 1992.

[2] Arras K.O., Vestli S.J ., "Hybrid, High-Precision Localization for the Mail Distributing Mobile Robot System MoPS", Proc. of the 1998 IEEE Int. Conf. on Robotics and Automation, p. 3134-3129, Leuven, Belgium, 1998.

[3] Arras K.O., Siegwart R.Y., "Feature Extraction and Scene Interpretation for Map-Based Navigation and Map Building", Proc. of SPIE, Mobile Robotics XII, Vol. 3210, p. 42-53, 1997.

[4] Astolfi A., "Exponential Stabilization of a Mobile Robot," Proc. of the Third European Control Conference, p. 3092-7, I taly, 1995.

[5] Bar-Shalom Y., Fortmann T.E., Tracking and Data Association, Mathematics in Science and Engineering, Vol. 179, Academic Press Inc., 1988.

[6] Brega R., "A Real-Time Operating System Designed for Predictability and Run-Time Safety", The Fourth I nt. Conference on Motion and Vibration Control (MOVIC'98), Zurich, Switzerland, 1998.

[7] Castellanos J .A., Tardós J .D., “Laser-Based Segmentation and Localization for a Mobile Robot", Proc. of the Sixth Int. Symposium on Robotics and Manufacturing (ISRAM'96), Montpellier, France, 1996.
[8] Chenavier F., Crowley J .L., "Position Estimation for a Mobile Robot Using Vision and Odometry", Proc. of the 1992 IEEE Int. Conference on Robotics and Automation, p. 2588-93, Nice, France, 1992.

[9] Crowley J.L., "World Modeling and Position Estimation for a Mobile Robot Using UItrasonic Ranging," Proc. of the 1989 IEEE Int. Conference on Robotics and Automation, $\mathrm{p}$. 674-80, Scottsdale, AZ, 1989.

[10] Delahoche L., Mouaddib E.M., Pégard C., Vasseur P., "A Mobile Robot Localization Based on a Multisensor Cooperation Approach," 22nd IEE E Int. Conference on Industrial Electronics, Control, and I nstrumentation (IECON'96), N ew York, USA, 1996.

[11] Leonard J .J ., Durrant-Whyte H.F., Directed Sonar Sensing for Mobile Robot Navigation, Kluwer Academic Publishers, 1992.

[12] Muñoz A.J ., Gonzales J., “Two-Dimensional Landmark-based Position Estimation from a Single I mage", Proc. of the 1998 IEEE Int. Conference on Robotics and Automation, Leuven, Belgium, 1998, p. 3709-14.

[13] Neira J., Tardos J .D., Horn J ., Schmidt G., "Fusing Range and I ntensity I mages for Mobile Robot Localization," IEEE Transactions on Robotics and Automation, 15(1):76-84, 1999.

[14] Pérez J .A., Castellanos J .A., Montiel J .M.M., Neira J . and Tardós J .D., "Continuous Mobile Robot Localization: Vision vs. Laser," Proc. of the 1999 IEEE Int. Conference on Robotics and Automation, Detroit, USA, 1999.

[15] Prescott B., McLean G.F., "Line-Based Correction of Radial Lens Distortion", Graphical Models and I mage Proc. 59(1), 1997, p. 39-47.

[16] Taylor R.M., Probert P.J ., "Range Finding and Feature Extraction by Segmentation of I mages for Mobile Robot Navigation", Proc. of the 1996 IEEE Int. Conference on Robotics and Automation, Minneapolis, USA, 1996. 